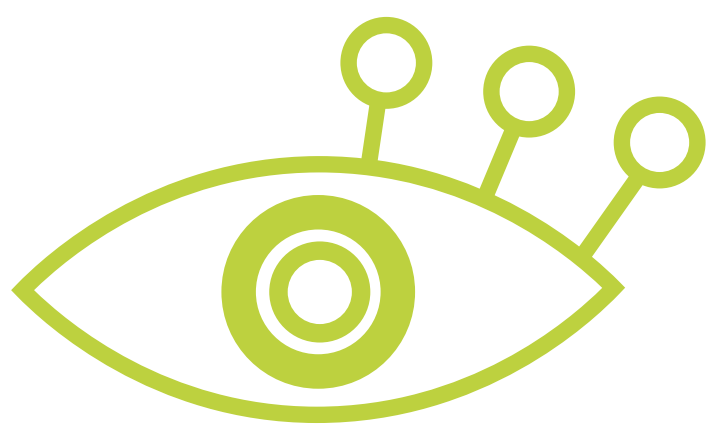

\title{
OLHO BIÔNICO PARA AUXÍLIO À LOCOMOÇÃO AUTONÔMA DE DEFICIENTES VISUAIS
}

BIONIC EYE FOR AIDS LOCOMOTION VISUALLY IMPAIRED

\section{Ricardo Alexandro de Medeiros Valentim}

Professor do Departamento de Engenharia Biomédica da Universidade Federal do Rio Grande do Norte, Doutor em Engenharia Elétrica e de Computação, Coordenador do Laboratório de Inovação Tecnológica em Saúde do Hospital Universitário Onofre Lopes. ricardo.valentim@ufrnet.br

\section{Pablo Holanda Cardoso}

Graduando em Engenharia da Computação da Universidade Federal do Rio Grande do Norte, Pesquisador do Laboratório de Inovação Tecnológica em Saúde do Hospital Universitário Onofre Lopes.

phol4nda@gmail.com

\section{Bruno Linhares de Souza}

Graduando no Bacharelado em Ciências e Tecnologia da Universidade Federal do Rio Grande do Norte, Pesquisador do Laboratório de Inovação Tecnológica em Saúde do Hospital Universitário Onofre Lopes. brunolinhares25000@gmail.com

Philippi Sedir Grilo de Morais

Mestre em Engenharia Elétrica e de computação, Pesquisador do Laboratório de Inovação Tecnológica em Saúde do Hospital Universitário Onofre Lopes.

philippi.sedir@gmail.com

\section{Antônio Higor Freire de Morais}

Professor de Sistemas de informação do Instituto Federal de Educação, Ciência e Tecnologia do Rio Grande do Norte, Mestre em Engenharia Elétrica e de computação, Pesquisador do Laboratório de Inovação

Tecnológica em Saúde do Hospital Universitário Onofre Lopes.

higor.morais@ifrn.edu.br

Rodrigo Dantas da Silva

Graduando em Engenharia da Computação da Universidade Federal do Rio Grande do Norte, Pesquisador do Laboratório de Inovação Tecnológica em Saúde do Hospital Universitário Onofre Lopes.

r_ds@hotmail.com

\section{João Victor Fernandes Cabral}

Graduando no Bacharelado em Ciências e Tecnologia da Universidade Federal do Rio Grande do Norte, Pesquisador do Laboratório de Inovação Tecnológica em Saúde do Hospital Universitário Onofre Lopes.

\section{jvictorcabral@hotmail.com}

\section{Hertz Wilton de Castro Lins}

Professor do Departamento de Engenharia de Comunicações da Universidade Federal do Rio Grande do Norte, Doutor em Engenharia Elétrica e de Computação, Pesquisador do Laboratório de Inovação

Tecnológica em Saúde do Hospital Universitário Onofre Lopes.

hertzw@gmail.com

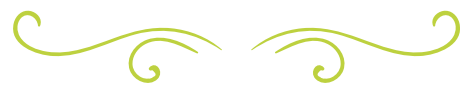




\section{RESUMO}

A grande demanda por soluções para reabilitação de pacientes com deficiência ou limitações visuais tem motivado o desenvolvimento das tecnologias assistivas. Estas tecnologias possibilitam o aumento da qualidade de vida dos pacientes através de uma maior autonomia no seu cotidiano. Este artigo apresenta uma solução de tecnologia assistiva aplicada na locomoção do paciente com deficiência visual.

PALAVRAS-CHAVE: Tecnologias assitivas, sistemas embarcados, locomoção autônoma.

\section{ABSTRACT}

The high demand for solutions for the rehabilitation of the patients with deficiency or visual impairment has motivated the development of the supportive technologies. This technologies enhances the life quality of the patients through a bigger daily autonomy. This article introduces an assistive technology solution into the locomotion of the visually impaired patient.

KEYWORDS: assistive technology, embedded systems, autonomous locomotion.

\section{INTRODUÇÃO}

No Brasil, segundo o censo realizado em novembro de 2010 pelo IBGE [1], existe um total de 35.774 .392 pessoas com alguma deficiência visual o que representa 18,75\% da população, Em todo o mundo, segundo a Organização mundial de Saúde, são estimados 285 milhões de pessoas com deficiência visual sendo 39 milhões cegas e 246 milhões com baixa visão. Neste cenário, a locomoção urbana para um deficiente visual pode ser uma tarefa desafiadora e com riscos potenciais de vida e acidentes, principalmente, pela grande quantidade e diversidade de obstáculos e da não adequação das estruturas como edificações, mobiliários, espaços e equipamentos as normas nacionais de qualidade de acessibilidade como, por exemplo, a ABNT 9050.

Em paralelo a este cenário, tecnologias móveis e soluções de sistemas embarcados são cada vez mais utilizadas em dispositivos e objetos como telefones, relógios, bicicletas, roteadores Wi-Fi e carros. Além destes, são observados também dispositivos que podem ser vestidos pelos usuários como óculos, anéis, pulseiras e trajes sendo estes exemplos da tecnologia conhecida como vestível [2]. Estas tecnologias possuem características como a capacidade de expansão da comunicação e interação do usuário com o mundo digital possibilitando uma grande diversidade de aplicações e benefícios como, por exemplo, o compartilhamento de informações entre usuários e dispositivos.

Neste contexto de possibilidades e convergências surge a motivação de viabilizar a locomoção urbana e autônoma de deficientes visuais com o desenvolvimento de soluções embarcadas ou vestíveis, sendo este desenvolvimento orientado para fornecer ao usuário um dispositivo com capacidade de adaptação as variações de cada trajeto facilitando e aumentando a segurança de sua locomoção gerando, desta forma, uma melhor qualidade de vida para o usuário, foram identificadas na literatura algumas soluções como os trabalhos de [3][4].

Este projeto apresenta uma arquitetura de hardware e software embarcado caracterizado como uma bengala inteligente eletrônica. A bengala foi desenvolvida para suportar a locomoção dos deficientes visuais e possui capacidades como reconhecer pessoas, objetos e identificar obstáculos na altura da cintura e da cabeça além de desníveis como buracos e degraus.

Outro recurso que possui a bengala é a capacidade de localização geográfica do usuário por outras pessoas interessadas sejam familiares ou cuidadores. Este recurso possui uma interface para acesso via internet, onde estes interessados podem obter acesso as seguintes funcionalidades para usuários e cuidadores: localização geográfica bengala, rota percorrida, visualização e 
histórico de obstáculos identificados e problemas de acessibilidade.

A identificação dos obstáculos é realizada através de sensores ultrassônicos localizados em várias partes como: punho da bengala, itens de chapelaria ou colar e na extremidade inferior. A partir da conexão do celular o usuário recebe sinas de alerta por comandos de voz previamente gravadas ou sinais sonoros customizados para informar o tipo de risco identificado, que pode ser um obstáculo ou desnível e a respectiva distância. Os alertas emitidos podem também ser vibratórios e diferentes para cada tipo de obstáculo viabilizando assim a utilização também por usuários com deficiência auditiva, caso não seja identificado nenhum obstáculo ou desnível a bengala informará que o trajeto está seguro através de alertas vibratórios ou sonoros.

Este artigo está organizado da seguinte forma: após a introdução são apresentados conceitos e exemplos de dispositivos de tecnologia assistiva desenvolvidos para a locomoção autônoma e em seguida é apresentada a especificação da arquitetura da bengala, finalizando na seção considerações finais é apresentada uma conclusão do trabalho.

\section{DISPOSITIVOS PARA LOCOMOÇÃO AUTÔNOMA}

Nas últimas décadas, segundo o trabaIho de [5], são observados alguns avanços e desafios em tecnologias assistivas aplicadas em dispositivos de locomoção, estas tecnologias segundo [4] podem ser classificadas em tecnologias para aprimoramento ou aumento, restituição e substituição da visão descritas a seguir:

As tecnologias de aprimoramento ou aumento da visão caracterizam-se pela integração de dispositivos como câmeras e o processamento da informação que é apresentada para visualização em equipamentos como: monitores. Tecnologias de restituição da visão caracterizam-se pela exibição da informação capturada por algum sensor e transmitida diretamente no córtex visual do cérebro humano ou através do nervo óptico.

O conceito principal utilizado nas tecnologias de substituição, é que a informação do ambiente fornecida pelos sensores é apresentada ao usuário em forma de sinais vibrotáteis, sendo estes sinais simultâneos ou não. Segundo [4], as tecnologias de substituição da visão podem ser classificadas em três subcategorias: ETAS (Electronic Travel AIDS), EOAs (Electronic Orientation AIDS) e PLDS (Position Locator Devices). São exemplos destas tecnologias $[6,7,8,18,19]$.

A partir da classificação das características citadas foram observadas algumas soluções baseadas em tecnologias assistivas para viabilizar a locomoção autônoma. Estas soluções possuem similaridades como: utilização de microcontroladores para integração e controle dos sensores e interface; Utilização de sensores ultrassônicos, dentre outros tipos; mobilidade e capacidade de emitir sinais de alerta sonoros ou vibráteis.

Voltando a [3], neste trabalho foi desenvolvido um dispositivo caracterizado como pulseira eletrônica com capacidade de identificar obstáculos acima da linha da cintura com a utilização de sensores ultrassônicos e sinais vibráteis. $O$ dispositivo possui um recurso, no qual possibilita ao usuário escolher o ajuste de acordo com o tipo de ambiente, aberto ou fechado, sendo o aberto para grandes distâncias, inferiores a 2 metros, e o fechado para pequenas distâncias, inferiores a 1 metro. O sistema possui um alerta vibratório, quando utilizado para pequenas distâncias, emitindo uma vibração alta e contínua, quando utilizado para grandes distâncias emite uma vibração que se intensifica conforme o usuário se aproxima do obstáculo detectado.

O dispositivo desenvolvido em [9], caracteriza-se como uma bengala e utiliza sensores e a câmera RGB do Microsoft kinect acoplados a um microcomputador e a uma bengala convencional para identificar obstáculos no trajeto, estes obstáculos são avisados ao usuário do dispositivo através de sinais sonoros.

O dispositivo apresentado no trabalho de [10], é caracterizado como bengala eletrônica, com capacidade de detectar obstáculos acima da cintura fornecendo uma resposta tátil ao usuário, através de um 
alerta vibratório no interior da bengala, aumentando gradativamente a frequência de vibração à medida que o usuário se aproxima do obstáculo. O sistema utiliza sensores ultrassônicos.

No trabalho de [11] foi desenvolvido um dispositivo caracterizado como cinto ultrassônico. $\bigcirc$ sistema utiliza sensores ultrassônicos acoplados a um cinto e um microcontrolador ATMEGA16A. Além disso, o dispositivo emite um alerta vibratório para alertar o usuário sobre os obstáculos.

Neste projeto [12], foi desenvolvida uma bengala eletrônica para auxílio na locomoção de deficientes visuais que utiliza sensores infravermelhos para detecção de obstáculos. Neste dispositivo caso seja detectado um obstáculo a uma distância maior do usuário ao objeto será disparado apenas um alerta vibratório curto, mas se o usuário estiver em perigo eminente, ou seja, muito próximo a algum objeto detectado pelo sensores o dispositivo irá disparar um alerta vibratório longo e contínuo.

Este trabalho [13] descreve uma pesquisa realizada sobre dispositivos utilizados no auxílio a deficientes visuais na locomoção em ambientes aberto. Com destaque, o primeiro sistema citado refere-se ao vOlCe, este sistema é composto por uma câmera de vídeo e um computador que transforma uma imagem em um mapeamento sonoro, as mensagens são enviadas direto para os auscultadores do usuário. Outro sistema citado é o sistema University of Stuttgart System que é composto por um computador de processamento e um módulo de sensor acoplado a bengala. Para aumentar o desempenho de detecção é construído um modelo 3D virtual do ambiente correspondente aos dados recebidos a partir do sensor.

Neste trabalho [14] foi desenvolvido o dispositivo caracterizado como luva sonar, este dispositivo utiliza dois pares de sensores ultrassônicos acoplados a um par de luvas. $\bigcirc$ sistema utiliza um microcontrolador Arduino. O princípio de funcionamento baseia-se nos mesmo princípio dos outros dispositivos citados, ao detectar um obstáculo é acionado um motor vibratório para alertar o usuário sobre a presença do obstáculo.
Neste trabalho [15] foi desenvolvido um dispositivo intitulado óculos sonar, este sistema utiliza sensores ultrassônicos acoplados a uns óculos. Os obstáculos podem ser detectados pelos sensores a uma distância mínima de $20 \mathrm{~cm}$ e máxima de $1,5 \mathrm{~m}$. $O$ sistema é dotado de um microcontrolador do tipo PIC e fones de ouvidos com um potenciômetro, quando um obstáculo é detectado um sinal sonoro é ativado que se intensifica conforme se aproxima do objeto.

Neste trabalho [16] foi desenvolvido um dispositivo intitulado bengala inteligente, este dispositivo utiliza redes neurais artificiais para detectar as distâncias do usuário ao obstáculo, levando em consideração a velocidade de deslocamento desse. O sistema possui sensores periféricos e um microcontrolador. Funciona utilizando-se o conceito de aprendizagem por reforço de acordo com o comportamento do usuário retardando ou antecipando o alerta em tempo real, diante disso, com o uso contínuo a bengala vai se adaptando mais ao padrão de comportamento do usuário e o seu uso tende a se tronar mais eficiente.

O dispositivo emite um alerta vibratório ao detectar algum obstáculo. Além disso, o sistema é acompanhado com um sensor de luminosidade HDR (High Dynamic Range), este aciona um LED ao perceber uma redução na luminosidade, auxiliando a pessoas e veículos a identificar o deficiente visual. Uma das principais desvantagens é o fato de elevar em muito o peso da bengala em relação ao peso da bengala branca convencional.

Neste trabalho [17] foi utilizado um dispositivo portátil para auxílio à locomoção de pessoas com deficiência visual utilizando um microcontrolador do tipo PIC para controlar sensores ultrassônicos posicionados nos ombros do usuário e na própria bengala. Além disso, o sistema é composto por dois vibradores, um acelerômetro e um pedal. A bengala possui uma roda para auxiliar aos deficientes visuais no reconhecimento dos terrenos no qual estão se locomovendo. A mensagem pode ser transmitida via sinal de alerta vibratório e comando de voz. 


\section{ARQUITETURA}

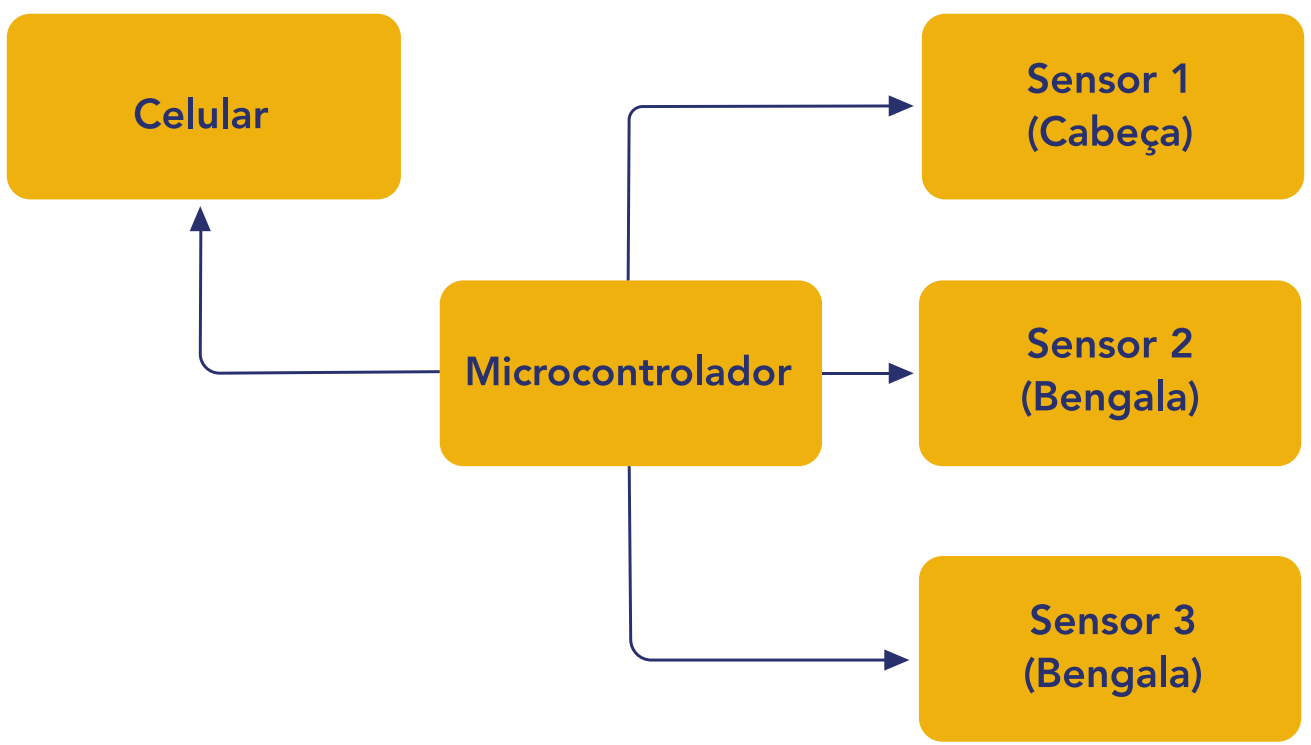

Figura 1 - Arquitetura.

A bengala eletrônica desenvolvida neste projeto utiliza uma arquitetura de um sistema embarcado, com características de vital importância para viabilidade do dispositivo, tais como: baixo custo, dissipação de potência reduzida, possibilita um processamento e uma resposta em tempo real. Tudo isso aliado à facilidade de acesso aos componentes para construção do dispositivo.

Neste dispositivo, são usados três sensoresultrassônicos para coletar as informações do ambiente. Os sensores ultrassônicos tem como princípio de funcionamento o sonar, no qual as ondas de ultrassom, inaudíveis para o ouvido humano, são emitidas e ao colidirem com algum obstáculo são imediatamente refletidas e captadas pelo receptor do sonar. Em seguida, estas informações são enviadas a unidade controladora do protótipo.

Na figura 1 é apresentada a arquitetura do dispositivo, o sensor 1 é responsável por detectar obstáculos acima da linha do tronco e da cabeça, este sensor deverá ser alocado na parte superior como por exemplo, itens de chapelaria, no colar ou em óculos. O sensor 2 deve detectar obstáculos acima da linha da cintura, este sensor fica localizado na empunhadura da bengala. $\bigcirc$ sensor 3, localizado na extremidade inferior da bengala, detecta desníveis no terreno como: buracos, bueiros, degraus e batentes.

Ao detectar um obstáculo ou desnível, o dispositivo irá reproduzir um alerta sintetizado de voz que se dá por meio de frases previamente gravadas ou por vibrações. As frases informam o tipo de perigo que se encontra à frente com as seguintes informações: obstáculo ou desnível, altura do obstáculo, e a distância desses. Caso seja um deficiente visual surdo, o dispositivo irá gerar alertas vibrotáteis diferentes para cada tipo de obstáculo ou desnível por meio de vibrações na própria bengala. Quando não houver mais obstáculos, ou desníveis, à frente, a bengala emitirá um alerta, sonoro ou vibratório, informando que o trajeto está seguro. Além disso, o dispositivo é capaz de reconhecer objetos ou pessoas e informar ao deficiente visual e calcular a velocidade com a qual o usuário está se aproximando do obstáculo. No dispositivo, o usuário pode optar por receber um dos tipos de informação, voz ou vibração, os dois ou nenhum.

O sistema de alimentação da bengala pode ser feito por meio de baterias recarregáveis ou não, permitindo a troca sem que 
o sistema seja desligado e ainda oferece um recurso de alerta ao usuário quando a bateria estiver com baixo nível de carga para que esta seja substituída. Também é possível alimentar o sistema através de uma fonte de alimentação externa.

\section{MÓDULO DE COMUNICAÇÃO SEM FIO}

Devido a necessidade de transmitir as informações do dispositivo diretamente ao celular de uma forma rápida e eficaz, a comunicação entre o dispositivo e o celular é realizada via bluetooth pelo protocolo IEEE: 802.15 com frequência $2,45 \mathrm{GHz}$ classe 2 com alcance de até $10 \mathrm{~m}$ e potência $2.5 \mathrm{~mW}$, diante disso, um baixo gasto de energia. Além disso, é possível a comunicação com mini-autofalantes pelo mesmo protocolo, que podem ser utilizado como um acessório ao dispositivo. A figura 2 apresenta a interface de comunicação em um celular.

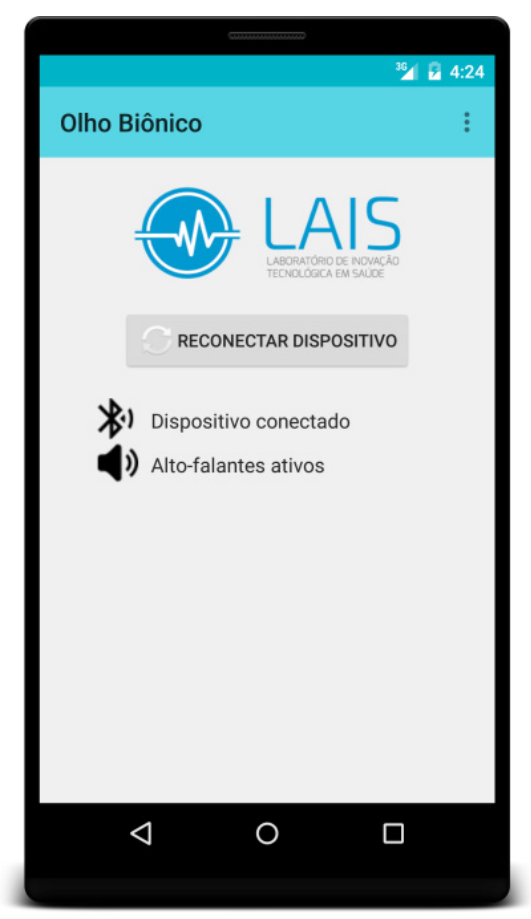

Figura 2 - Interface da aplicação Web.

\section{SISTEMA DE GEORREFERENCIAMENTO}

A bengala do projeto olho biônico possui também um sistema de denúncia para georreferenciamento de algum obstáculo ou qualquer outro problema de acessibilidade que não estejam em conformidade com as vias públicas, por exemplo, buracos e caixas de aparelhos condicionadores de ar aparente nas paredes. Além disso, 0 dispositivo também possui como recurso um sistema GPS (Geo Position System), que possibilita aos familiares ou cuidadores do deficiente visual obter a localização geográfica ou a rota executada por ele. A figura 3 apresenta o sistema de georeferenciamento exemplificando obstáculos identificados pelo usuário. 


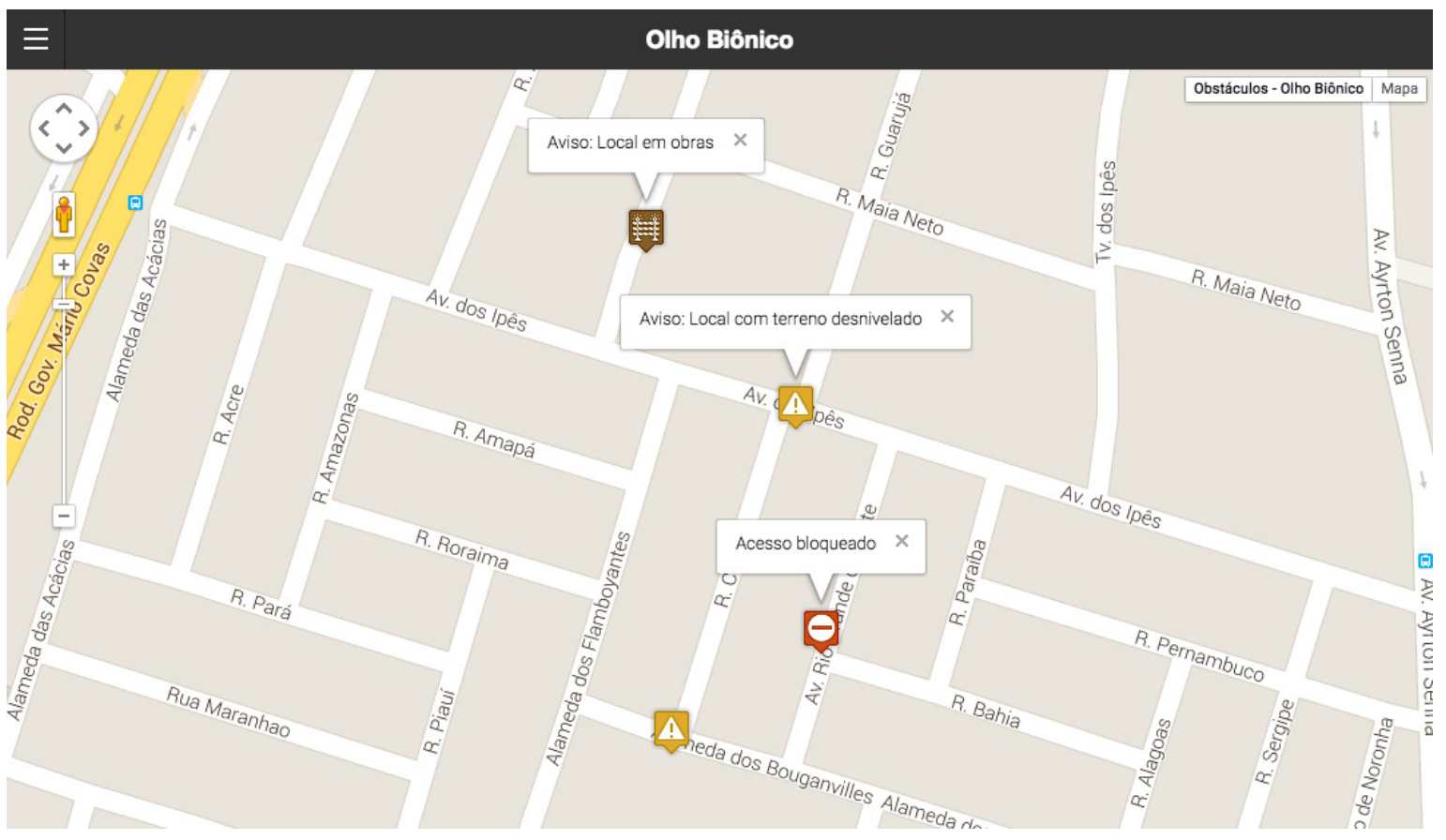

Figura 3 - Sistema de Georreferenciamento

\section{CONCLUSÃO}

O projeto olho biônico apresentado neste trabalho auxilia pessoas com deficiência visual a se locomoverem com mais segurança em vias públicas ou ambientes fechados como: casas, prédios, teatros e etc., possibilitando a detecção de obstáculos comumente encontrados nesses locais. Este projeto é registrado como uma patente brasileira com o Título: Bengala inteligente para auxílio à locomoção de deficientes visuais e com o registro: BR 2020140221277 . (c) 


\section{REFERÊNCIAS}

IBGE. Censo Demográfico 2010, Instituto Brasileiro de Geografia e Estatística, 2013.

OLSSON, T.A. Wearables. technology in action. ed. springer. 2012.

FILHO, J.S.R., CALVACANTE, R.V, NATASHA, A., BARROS, R. ARGOS. Auxílio à locomoção de deficientes visuais a partir de pulseira microcontrolada. Computer on the Beach, p. 89-98, 2011.

DAKOPOULOS, D., BOURBAKIS, N.G. Wearable obstacle avoidance electronic travel aids for blind: a survey. IEEE

Transactions on Systems, Man, and Cybernetics, v. 40, n.1, p. 2535, Jan. 2010.

BLASCH, B. B., WIENER W. R., Welsh, R. L. Foundations of

Orientation and Mobility, 2nd ed. New York: AFB Press, 1997.

IFUKUBE, T., SASAKI, T., PENG, C. A blind mobility aid modeled after echolocation of bats. IEEE Transactions Biomedical

Engineering , v. 38, n. 5, p. 461-465, May 1991.

SHOVAL, S., BORENSTEIN, J., KOREN, Y. Mobile robot obstacle avoidance in a computerized travel aid for the blind. Robotics and Automation. IEEE, p. 2023-2028, 1994.

MEIJER, Peter BL. An experimental system for auditory image representations. IEEE Transactions on Biomedical Engineering, $\mathrm{v}$. 39, n. 2, p. 112-121, Feb. 1992.

KIST, G., BAGGIO, M. A., SILVA, F.L., JOAQUIM, J.R., LIMA, L.P. PADEVI-Protótipo de Auxilio a Deficientes Visuais. Revista de Empreendedorismo, Inovação e Tecnologia, v. 1, n. 1, p. 45-57, 2014.

FONSECA, R., GARCÍA, A.R., DURÁN, A. Electronic long cane for locomotion improving on visual impaired people. A case study. Pan American Health Care Exchanges (PAHCE), p.58-61, April 2011.

RAGHUVANSHI, D.S., DUTTA, I., VAIDYA, R.J. Design and analysis of a novel sonar-based obstacle-avoidance system for the visually impaired and unmanned systems. International conference on Embedded Systems (ICES), p.238-243, July 2014.

INNET, S., RITNOOM, N. An application of infrared sensors for electronic white stick. Intelligent Signal Processing and Communications Systems, 2008. IEEE, p. 1-4, 2009. 
TAPU, R. MOCANU, B., TAPU, E. A survey on wearable devices used to assist the visual impaired user navigation in outdoor environments. Electronics and Telecommunications (ISETC), 11th International Symposium on. IEEE, p. 1-4, 2014.

VILLALBA, E. S., PEIXOTO, A. E.F., SOUZA, L.F., BELLÓ, V. F Luva Sonar-Dispositivo de auxílio a mobilidade para deficientes visuais. Computer on the Beach, p. 546-547, 2015.

GONZATTO, A., SANTOS, C., MELO, F., FARIA, J.R., KONIGAME, E.M. Óculos sonar para deficientes Visuais. XIII Encontro Latino Americano de Iniciação Científica e IX Encontro Latino Americano de Pós-Graduação - Universidade do Vale do Paraíba, 2012.

ALVES, F. A.S., NEUMANN, A. M.M., GOUVÊA JR, M. M. Bengala Inteligente Neural Baseada em Aprendizagem por Reforço para Deficientes Visuais. Disponível em: <http://www.lbd.dcc.ufmg.br/ colecoes/eniac/2014/0071.pdf>. Acesso em: 20 jul 2014.

BOUSBIA-SALAH, M., BETTAYEB, M., LARBI, A. A Navigation Aid Blind People. Journal of Intelligent \& Robotic Systems, V. 64, n. 3-4, p. 387- 400, May 2011.

HUB, A., DIEPSTRATEN, J., ERTL, T. Design and development of an indoor navigation and object identification system for the blind. ACM SIGACCESS Accessibility Computing, n. 77-78, p. 147-152, Sep. 2003/Jan. 2004.

AGUERREVERE, D., CHOUDHURY, M., BARRETO, A. Portable 3D sound / sonar navigation system for blind individuals. Second LACCEI International Latin American and Caribbean Conference for Engineering and Technology (Miami), Jun. 2004. 